\title{
Dirichlet boundary value problems of the Ernst equation
}

\author{
Marcus Ansorg, Andreas Kleinwächter, Reinhard Meinel, and Gernot Neugebauer \\ Theoretisch-Physikalisches Institut, University of Jena, Max-Wien-Platz 1, 07743 Jena, Germany
}

(Received 28 September 2001; published 11 January 2002)

\begin{abstract}
We demonstrate how the solution to an exterior Dirichlet boundary value problem of the axisymmetric, stationary Einstein equations can be found in terms of generalized solutions of the Bäcklund type. The proof that this generalization procedure is valid is given, which also proves conjectures about earlier representations of the gravitational field corresponding to rotating disks of dust in terms of Bäcklund-type solutions. As a further result, we find that, in contrast with the Laplace equation, arbitrary boundary values may not be prescribed.
\end{abstract}

DOI: 10.1103/PhysRevD.65.044006

PACS number(s): 04.20.Jb, 04.25.Dm

\section{INTRODUCTION}

Extraordinarily massive, compact astrophysical objects such as neutron stars require a fully relativistic treatment. This motivates the study of the relativistic gravitational field of axisymmetric, stationary rotating bodies.

The investigation of the exterior field, i.e. of arbitrary exterior boundary value problems of the axisymmetric stationary Einstein equations, can only be a first step in this direction, for neither the shape of the body (i.e. the boundary) nor particular boundary values may be prescribed. For a given equation of state characterizing the matter of the rotating body, the boundary and the values there result from transition conditions since the metric along with its first normal derivative behave continuously at the boundary (in appropriate coordinates). Therefore, a subsequent step must be the combination of a procedure to compute the interior solution with the method to treat the exterior field in order to realize the transition conditions.

Our aim is to show a way to handle the exterior field. In particular, we want to make use of the variety of exact analytic solutions that are available in the exterior region. Thanks to the facts that (1) the exterior vacuum field equations can be summarized in a single nonlinear complex differential equation-the so-called Ernst equation [1,2], and (2) the Ernst equation has been found to be the integrability condition of a corresponding linear matrix problem, it is possible to apply various soliton methods (see [3-13]) to create explicit exterior solutions, among them the Bäcklund transformations and the Riemann-Hilbert techniques. By extensive investigation of the latter, Neugebauer and Meinel succeeded in solving the boundary value problem that corresponds to an infinitesimal thin rigidly rotating disk of dust [14-16]. In particular, they found their solution to belong to a "hyperelliptic class" of solutions [17], see also [18-21]. Further investigations as to whether this class of solutions can be used to solve more general boundary value problems corresponding to differentially rotating disks of dust have been carried out by Ansorg and Meinel [22] and by Ansorg [23]. In the latter paper it was demonstrated that the
Bäcklund type solutions ${ }^{1}$ suffice to approximate the exterior gravitational field of an arbitrary differentially rotating disk of dust.

In this paper we investigate the question as to whether the treatment of the boundary value problem of an arbitrary differentially rotating disk of dust by means of Bäcklund type solutions can also be applied to extended boundaries $B$, which can potentially form the shape of a rotating body. We denote by $B$ a smooth spatial curve, at which regular boundary values are prescribed. The solubility of such Dirichlet boundary value problems for sufficiently weak relativistic boundary values has been proven by Reula [24], see also $[25,26]$. In illustrative examples we will take spherical boundaries in order to make the formulas more transparent and therefore get better insight into the underlying mathematical structure. However, the general statements are valid for arbitrary extended $B$.

The paper is organized as follows. At first, the metric tensor and the Ernst equation are introduced. Then, solution techniques to solve boundary value problems of the real axisymmetric three-dimensional Laplace equation with the boundary $B$ are discussed in order to prepare the relativistic treatment. These solutions will be given in terms of an analytic function $H$, which is defined on a curve $\Gamma$ in the complex plane and satisfies $H(\bar{X})=\overline{H(X)}$ (with $X \in \mathrm{C}$ and the bar denoting complex conjugation). Here, the curve $\Gamma$ is closely connected with the boundary $B$. In this formulation, the solutions describe both a regular interior and exterior field, which (in general) assumes different values on the inner and outer side of $B$. The prescription of these interior and exterior boundary values uniquely determines the function $H$, i.e. this formulation permits the simultaneous solution of an interior and exterior boundary value problem.

The second section treats the hyperelliptic solutions of the Ernst equation and their generalization by means of a suitable limiting process. Similar to the formulation of solutions

\footnotetext{
${ }^{1}$ The Bäcklund type solutions can be obtained by applying Bäcklund transformations to purely real "seed" solutions of the Weyl class. These solutions form a subclass of the hyperelliptic class; see [23].
} 
of the real Laplace equation, we find these generalized solutions (1) to depend on an analytic function $\gamma$ defined on $\Gamma$ (and, in general, not subject to further requirements) and (2) to permit the simultaneous solution of an interior and exterior Dirichlet boundary value problem of the Ernst equation. In the case of a flat interior field we find an explicit relation of the function $\gamma$ and the values of the Ernst potential at the exterior part of the symmetry axis.

In Sec. III A, we investigate the solutions of the Bäcklund type. Apart from regularity properties we discuss the generalization of these solutions which can be performed since they form a subclass of the hyperelliptic solutions. As a result we prove conjectures formulated in [23], in particular that the Neugebauer-Meinel solution can be written as a well defined limit of Bäcklund type solutions.

Because of their mathematical simplicity (compared with the much more complicated hyperelliptic solutions), the Bäcklund type solutions play the fundamental role in our treatment for approximating solutions of an exterior boundary value problem by analytic solutions. This is carried out in Sec. III A. As a result, we find that in contrast to the Laplace equation, arbitrary boundary values of the Dirichlet type may not be prescribed.

In what follows, units are used in which the velocity of light as well as Newton's constant of gravitation are equal to 1 .

\section{A. Metric tensor, Ernst equation, and boundary conditions}

The metric tensor for axisymmetric stationary and asymptotically flat vacuum space-times reads as follows in WeylPapapetrou-coordinates $(\rho, \zeta, \varphi, t)$ :

$$
d s^{2}=e^{-2 U}\left[e^{2 k}\left(d \rho^{2}+d \zeta^{2}\right)+\rho^{2} d \varphi^{2}\right]-e^{2 U}(d t+a d \varphi)^{2} .
$$

The field equations are equivalent to a single complex equation - the so-called Ernst equation

$$
\begin{aligned}
(\Re f) \Delta f & =(\nabla f)^{2}, \\
\Delta & =\frac{\partial^{2}}{\partial \rho^{2}}+\frac{1}{\rho} \frac{\partial}{\partial \rho}+\frac{\partial^{2}}{\partial \zeta^{2}}, \quad \nabla=\left(\frac{\partial}{\partial \rho}, \frac{\partial}{\partial \zeta}\right),
\end{aligned}
$$

where the Ernst potential $f$ is given by

$$
f=e^{2 U}+\mathrm{i} b \quad \text { with } \quad b_{, \zeta}=\frac{e^{4 U}}{\rho} a_{, \rho}, \quad b_{, \rho}=-\frac{e^{4 U}}{\rho} a_{, \zeta} .
$$

The remaining function $k$ can be calculated from the Ernst potential $f$ by a line integral:

$$
\begin{aligned}
& \frac{k_{, \rho}}{\rho}=\left(U_{, \rho}\right)^{2}-\left(U_{, \zeta}\right)^{2}+\frac{1}{4} e^{-4 U_{[}}\left[\left(b_{, \rho}\right)^{2}-\left(b_{, \zeta}\right)^{2}\right], \\
& \frac{k_{, \zeta}}{\rho}=2 U_{, \rho} U_{, \zeta}+\frac{1}{2} e^{-4 U_{, \rho} b_{, \zeta} .}
\end{aligned}
$$

In this paper we treat the following boundary value problem of the Ernst equation (1):
(1) The boundary $B$ is given by a smooth spatial curve which can be described by some positive analytic function $r_{B}:[-1,1] \rightarrow \mathrm{R}_{+}:$

$$
\begin{aligned}
B= & \left\{(\rho, \zeta): \rho=r_{B}(\cos \vartheta) \sin \vartheta, \zeta=r_{B}(\cos \vartheta) \cos \vartheta,\right. \\
& \vartheta \in[0, \pi]\} .
\end{aligned}
$$

(2) Along the boundary $B$ we require regular boundary conditions of the Dirichlet type, i.e. $f$ is given for $(\rho, \zeta) \in B$.

(3) Regularity at the rotation axis $\rho=0$ is guaranteed by

$$
\frac{\partial f}{\partial \rho}(0, \zeta)=0 .
$$

(4) At infinity, asymptotic flatness is realized by $f \rightarrow 1$.

The treatment in Sec. II will provide us a formulation $f$ $=f(\gamma)$ which is sufficiently general to satisfy interior and exterior boundary conditions of the Ernst equation (1), i.e. for an appropriate prescription ${ }^{2}$ of (not necessarily coinciding) interior and exterior boundary values, there is a uniquely determined function $\gamma: \Gamma \rightarrow \mathrm{C}$ [with $\Gamma=\{X \in \mathrm{C}:(|\Im X|, \mathfrak{\Re} X)$ $\in B\}]$ such that the Ernst potential $f=f(\gamma)$ is both regular within and without $B$ and assumes the above boundary values at the inner and outer side of $B$. Since for physical reasons we are only interested in an exterior solution, the freedom in the choice of the interior boundary values will be used to restrict the function $\gamma$ such that the solution $f$ $=f(\gamma)$ can be represented by Bäcklund type solutions. This is outlined in the third section. Furthermore, for the exterior solution we will impose the additional physical requirement of reflectional symmetry with respect to the plane $\zeta=0$ (see [27]) which leads to

$$
\begin{aligned}
r_{B}(\tau) & =r_{B}(-\tau) \text { for all } \tau \in[-1,1], \\
f(\rho,-\zeta) & =\overline{f(\rho, \zeta)} \text { for all }(\rho, \zeta) \text { outside } B .
\end{aligned}
$$

\section{B. Boundary value problems of the Laplace equation}

In this section we prepare the general formulation $f$ $=f(\gamma)$ by considering the corresponding non-relativistic gravitational boundary value problem-i.e. the general boundary value problem of the axisymmetric threedimensional Laplace equation

$$
\Delta U=0 .
$$

Any real solution $U$ which is both regular inside and outside the boundary $B$ can be written in the form

$$
U(\rho, \zeta ; H)=\frac{1}{2 \pi \mathrm{i}} \oint_{\Gamma} \frac{H(X) d X}{W_{z}}
$$

with

\footnotetext{
${ }^{2}$ As already mentioned above, the Dirichlet boundary values cannot be chosen arbitrarily.
} 


$$
\Gamma=\{X \in \mathrm{C}:(|\Im X|, \mathfrak{R} X) \in B\},
$$

$H: \Gamma \rightarrow \mathrm{C}$ is an analytic function with $H(\bar{X})=\overline{H(X)}$,

$$
\begin{gathered}
W_{z}=\sqrt{(X-\zeta)^{2}+\rho^{2}}, \\
\mathfrak{R}\left(W_{z}\right)= \begin{cases}<0 & \text { for }(\rho, \zeta) \text { outside } B \text { or } \mathfrak{R}(X)<\zeta, \\
>0 & \text { for }(\rho, \zeta) \text { inside } B \text { and } \mathfrak{R}(X)>\zeta .\end{cases}
\end{gathered}
$$

The function $H$ is uniquely determined by the interior and exterior boundary values of the potential $U$. For a spherical boundary (with radius $r_{B} \equiv 1$ ) we may write $H$ in the form

$$
H(X)=\sum_{j=1}^{\infty}\left(H_{j}^{(+)} X^{j-1}+H_{j}^{(-)} X^{-j}\right), \quad H_{j}^{( \pm)} \in \mathbb{R} .
$$

Then the coefficients $H_{j}^{( \pm)}$can be read from an expansion of the boundary values in Legendre polynomials

$$
P_{n}(x)=\frac{1}{2^{n} n !} \frac{d^{n}}{d x^{n}}\left[\left(x^{2}-1\right)^{n}\right]
$$

of the cosine of the angular coordinate $\vartheta$ with $\rho$ $=r \sin \vartheta, \zeta=r \cos \vartheta$ :

$$
\begin{aligned}
& \lim _{r \uparrow 1} U(\rho, \zeta ; H)=\sum_{j=1}^{\infty} H_{j}^{(+)} P_{j-1}(\cos \vartheta) \\
& \lim _{r \downarrow 1} U(\rho, \zeta ; H)=-\sum_{j=1}^{\infty} H_{j}^{(-)} P_{j-1}(\cos \vartheta) .
\end{aligned}
$$

\section{Discussion}

(1) Reflectional symmetry

$$
U(\rho,-\zeta ; H)=\left\{\begin{array}{l}
\kappa U(\rho, \zeta ; H) \text { for }(\rho, \zeta) \text { within } B, \\
-\kappa U(\rho, \zeta ; H) \text { for }(\rho, \zeta) \text { without } B
\end{array}\right.
$$

with $\kappa^{2}=1$ is obtained if $r_{B}(\tau)=r_{B}(-\tau)$ and $H(-X)$ $=\kappa H(X)$, i.e. an odd (even) function $H$ produces an even (odd) exterior potential $U$ in the coordinate $\zeta$.

(2) The freedom of the choice of the interior field can be used to restrict the function $H$ in an appropriate manner. For example we may choose $\mathfrak{R}(H)=$ const for $X \in \Gamma$, where this constant can be arbitrarily prescribed. For the above spherical case, this results in $H_{j+1}^{(+)}=-H_{j}^{(-)}$. Then the function $H$ is uniquely determined only by the exterior boundary values. Analogously, in the relativistic treatment (see Sec. III B) we may choose the corresponding function $\gamma$ such that $\Im(\gamma)$ $=$ const $\neq 0$ for $X \in \Gamma$, which ensures that the corresponding Ernst potential $f$ can be approximated very well by solutions of the Bäcklund type.

\section{THE HYPERELLIPTIC CLASS OF SOLUTIONS AND ITS GENERALIZATION BY A SUITABLE LIMITING PROCESS}

Meinel and Neugebauer [17] as well as Korotkin [18,19], (see also [20,21]), were able to construct the hyperelliptic class of solutions containing a finite number of complex parameters and one arbitrary real solution to the axisymmetric three-dimensional Laplace equation. By investigation of corresponding Riemann-Hilbert problems one finds that this class can be generalized in an appropriate manner. The generalized class turns out to contain all Ernst potentials which are both regular within and without the boundary $B$, which assume (in general) different boundary values at the inner and outer side of $B$, and are sufficiently weakly relativistic, i.e. close to the flat space solution $f \equiv 1$. In this section we discuss these generalized solutions which allow us to approximate the solution to a simultaneous interior and exterior boundary value problem.

\section{A. The hyperelliptic class of solutions}

We adapt the hyperelliptic class of solutions as given in [17] to our purposes by writing: For a given integer $p \geqslant 1$, a $\operatorname{set}^{3}\left\{X_{1}, \ldots, X_{p}\right\}=\left\{X_{\nu}\right\}_{p}$ of complex parameters, and an analytic function $H: \Gamma \rightarrow \mathrm{C}, H(\bar{X})=\overline{H(X)}$, the following expression:

$$
\begin{aligned}
f\left(\rho, \zeta ;\left\{X_{\nu}\right\}_{p}, H\right)= & \exp \left(\sum_{\nu=1}^{p} \int_{X_{\nu}}^{X^{(\nu)}} \frac{X^{p} d X}{W}\right. \\
& \left.+\frac{1}{2 \pi \mathrm{i}} \oint_{\Gamma} \frac{H(X) X^{p} d X}{W_{z}}\right)
\end{aligned}
$$

with

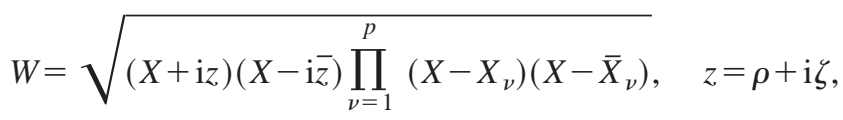

$$
\sum_{\nu=1}^{p} \int_{X_{\nu}}^{X^{(\nu)}} \frac{X^{j} d X}{W}=-\frac{1}{2 \pi \mathrm{i}} \oint_{\Gamma} \frac{H(X) X^{j} d X}{W_{z}}, \quad 0 \leqslant j<p
$$

satisfies the Ernst equation. The ( $z$-dependent) values for the $X^{(\nu)}$ as well as the integration paths on a two-sheeted Riemann surface have to be taken from the solution to the Jacobian inversion problem (7).

The Ernst potential ${ }^{4} f=f\left(\left\{X_{\nu\}_{p}}, H\right)\right.$ is regular at $(\rho, \zeta)$ $=\left(\left|\Im\left[X_{\nu}\right]\right|, \Re\left[X_{\nu}\right]\right)$ as can be deduced from an appropriate combination of the equations (7) and corresponding rearrangements of the terms occurring in Eq. (6). In general, $f\left(\left\{X_{\nu}\right\}_{p}, H\right)$ assumes different values at the inner and outer sides of the boundary $B$, i.e. it possesses a jump along $B$.

\footnotetext{
${ }^{3}$ In the following, the notation $\left\{X_{1}, \ldots, X_{p}\right\}$ will be abbreviated by $\left\{X_{\nu}\right\}_{p}$.

${ }^{4}$ For simplicity, we suppress the coordinate dependence and write only $f=f\left(\left\{X_{\nu}\right\}_{p}, H\right)$.
} 


\section{B. The generalization of the hyperelliptic class of solutions by a suitable limiting process}

This section contains a theorem which ensures that the above hyperelliptic solutions can be generalized in an appropriate manner such that simultaneous interior and exterior boundary value problems become soluble by means of these solutions. The theorem consists of three parts, the proofs of which are outlined. Moreover, we illustrate certain properties of the generalized class.

\section{Theorem}

Given the analytic function $\gamma: \Gamma \rightarrow \mathrm{C}$ which can be extended to some compact neighborhood $\mathrm{G}_{\gamma}$ about $\Gamma$, then

(1) for sufficiently small $\varepsilon$, the Ernst potential

$$
f(\varepsilon \gamma)=\lim _{p \rightarrow \infty} f\left(\left\{X_{\nu}^{(p)}\right\}_{p}, \varepsilon H_{p}\right)
$$

exists and is independent of the particular choice of the sequences $\left\{\left\{X_{\nu}^{(p)}\right\}_{p}\right\}_{p_{0}}^{\infty}$ and $\left\{H_{p}\right\}_{p_{0}}^{\infty}$ which serve to represent $\gamma$ by

$$
\gamma(X)=\lim _{p \rightarrow \infty} H_{p}(X) \prod_{\nu=1}^{p}\left(X-X_{\nu}^{(p)}\right) \quad \text { for } \quad X \in G_{\gamma} .
$$

(2) The Ernst potential $f=f(\varepsilon \gamma)$ is both regular inside and outside the boundary $B$ and assumes (in general) different boundary values at the inner and outer side of $B$. The exterior and interior Ernst potentials can be extended beyond the boundary $B$ to the region $\mathbb{B}_{\gamma}=\left\{(\rho, \zeta): \zeta \pm \mathrm{i} \rho \in \mathbb{G}_{\gamma}\right\}$.

(3) Any sufficiently weak relativistic Ernst potential $f$ which is both regular within and without the boundary $B$ with (in general) different boundary values at the inner and outer side of $B$, can be written in the above manner as $f$ $=f(\gamma)$. The function $\gamma$ is uniquely determined by the Dirichlet boundary values of $f$ at $B$.

\section{Proof}

(1) In order to prove the first one of the above statements we establish a relation between the function $\gamma$ and holomorphic functions $\alpha$ and $\beta$ defined on $\mathbb{G}_{\gamma}$ from which the Ernst potential $f$ can be calculated via the solution of a RiemannHilbert problem.

(a) To introduce $\alpha$ and $\beta$, we follow the treatments of [14,28] and [29]:

The Ernst equation is the integrability condition of the linear problem

$$
\begin{gathered}
\Phi_{, z}=[f+\bar{f}]^{-1}\left(\begin{array}{cc}
\bar{f}_{, z} & \lambda \bar{f}_{, z} \\
\lambda f_{, z} & f_{, z}
\end{array}\right) \Phi \\
\Phi_{, z}=[f+\bar{f}]^{-1}\left(\begin{array}{cc}
\bar{f}_{, \bar{z}} & \lambda^{-1} \bar{f}_{, \bar{z}} \\
\lambda^{-1} f_{, \bar{z}} & f_{, \bar{z}}
\end{array}\right) \Phi
\end{gathered}
$$

where $\Phi=\Phi(X, \rho, \zeta)$ is a $2 \times 2$-matrix and the spectral parameter $\lambda$ is given by

$$
\lambda(X)=\sqrt{\frac{X-\mathrm{i} \bar{z}}{X+\mathrm{i} z}} .
$$

One explicit way to create solutions to the Ernst equation that are regular within and without $B$ and possess a jump at $B$ is to use the Riemann-Hilbert-techniques, by which we force the corresponding matrix $\Phi$ to possess a multiplicative jump at $\Gamma$ :

$$
\Phi_{+}=\Phi_{-} C(X)
$$

and moreover to be regular within and without $\Gamma$. The indices "+" and "- " refer to the interior and exterior sides of $\Gamma$, respectively. The jump-matrix $C=C(X)$ is independent of the coordinates $(z, \bar{z})$ and can be cast into the form

$$
C(X)=\left(\begin{array}{ll}
\alpha(X) & 0 \\
\beta(X) & 1
\end{array}\right), \quad \overline{\alpha(X)}=\alpha(\bar{X}), \quad \overline{\beta(X)}=-\beta(\bar{X})
$$

If the functions $\alpha$ and $\beta$ are prescribed, the Ernst potential at an arbitrary point $(\rho, \zeta)$ can be determined by the solution of a linear integral equation; see [28].

As outlined in [29], the linear system can be integrated along the rotation axis, which yields in agreement with the above Rieman-Hilbert problem $\left[\rho=0, \zeta>-r_{B}(-1)\right]$ :

$$
\begin{aligned}
& \Phi^{+}=\left(\begin{array}{cc}
\bar{f} & 1 \\
f & -1
\end{array}\right)\left(\begin{array}{ll}
F(X) & 0 \\
B(X) & 1
\end{array}\right) \\
& \Phi^{-}=\left(\begin{array}{cc}
1 & \bar{f} \\
1 & -f
\end{array}\right)\left(\begin{array}{cc}
1 & B(X) \\
0 & F(X)
\end{array}\right) .
\end{aligned}
$$

The notation $\Phi^{ \pm}$refers to the two sheets on which $\Phi$ is defined; " \pm " means the sheet in which $\lambda= \pm 1$ for $\rho=0$. The functions ${ }^{5} F=F(X)$ and $B=B(X)$ are defined in the complex plane and are regular within and without the contour $\Gamma(F \rightarrow 1$ and $B \rightarrow 0$ as $|X| \rightarrow \infty)$ but possess a jump at $\Gamma$. This jump can be taken from the above Riemann-Hilbert problem:

$$
F_{+}=\alpha F_{-}, \quad B_{+}=\alpha B_{-}+\beta
$$

(b) We establish a connection between the relativistic parameter $\varepsilon$ as well as $\gamma$ and the functions $F_{ \pm}$and $B_{ \pm}$, now additionally depending on $\varepsilon$. Thus, by virtue of Eq. (10), we get $\alpha=\alpha(X ; \varepsilon ; \gamma)$ and $\beta=\beta(X ; \varepsilon ; \gamma)$.

Given the function $\gamma$ which is holomorphic on $\mathrm{G}_{\gamma}$, we denote by $\mathcal{B}_{\gamma}$ the Banach space of all holomorphic functions on $G_{\gamma}$ with the norm

$$
\|\sigma\|=\sup _{X \in \mathrm{G}_{\gamma}}|\sigma(X)| \quad \text { for } \quad \sigma \in \mathcal{B}_{\gamma} .
$$

We now define functions

\footnotetext{
${ }^{5}$ The function $B=B(X)$ is not to be confused with the boundary
} $B$. 


$$
F_{ \pm}:\left[0, \varepsilon_{\gamma}\right] \rightarrow \mathcal{B}_{\gamma}, \quad B_{ \pm}:\left[0, \varepsilon_{\gamma}\right] \rightarrow \mathcal{B}_{\gamma}
$$

that satisfy the following system of differential equations:

$$
\begin{gathered}
\varepsilon \frac{d}{d \varepsilon} F_{ \pm}=F_{ \pm}\left(\hat{L}_{ \pm} s_{1}\right)+B_{ \pm}\left(\hat{L}_{ \pm} s_{2}\right) \\
2 \varepsilon \frac{d}{d \varepsilon} B_{ \pm}=\frac{B_{ \pm}^{2}-1}{F_{ \pm}}\left(\hat{L}_{ \pm} s_{2}\right)+F_{ \pm}\left(\hat{L}_{ \pm} s_{3}\right) .
\end{gathered}
$$

Here, the $s_{j}$ as well as the $\hat{L}_{ \pm}$are introduced as follows:

(i) The $s_{j}, s_{j}:\left[0, \varepsilon_{\gamma}\right] \rightarrow \mathcal{B}_{\gamma}$, follow from $F_{ \pm}, B_{ \pm}$by

$$
\begin{gathered}
{\left[\left(F_{+}+F_{-}\right) s_{1}+\left(B_{+}+B_{-}\right) s_{2}\right] \tanh \frac{G}{2}=G\left(F_{-}-F_{+}\right)} \\
{\left[\left(B_{+}+B_{-}\right) s_{1}+\left(\frac{B_{+}^{2}-1}{F_{+}}+\frac{B_{-}^{2}-1}{F_{-}}\right) s_{2}\right] \tanh \frac{G}{2}} \\
=G\left(B_{-}-B_{+}\right) \\
s_{3}=F_{+}^{-2}\left[2 F_{+} B_{+} s_{1}+\left(B_{+}^{2}-1\right) s_{2}\right] \\
G^{2}=s_{1}^{2}+s_{2} s_{3} .
\end{gathered}
$$

(ii) Any function $\sigma \in \mathcal{B}_{\gamma}$ can uniquely be written as the sum

$$
\sigma=\sigma_{+}+\sigma_{-}
$$

with $\sigma_{+}$and $\sigma_{-}$only possessing singularities without and within the curve $\Gamma$, respectively, and $\sigma_{-} \rightarrow 0$ as $|X| \rightarrow \infty$. The linear operators $\hat{L}_{ \pm}, \hat{L}_{ \pm}: \mathcal{B}_{\gamma} \rightarrow \mathcal{B}_{\gamma}$, extract these functions $\sigma_{ \pm}$:

$$
\hat{L}_{ \pm} \sigma=\mp \sigma_{ \pm} .
$$

Explicitly:

$$
\begin{aligned}
\frac{1}{2 \pi \mathrm{i}} \oint_{\Gamma} \frac{\sigma(Y) d Y}{X-Y} \\
\quad=\left\{\begin{array}{l}
\left(\hat{L}_{+} \sigma\right)(X)=-\sigma_{+}(X) \text { for } X \text { within } \Gamma, \\
\left(\hat{L}_{-} \sigma\right)(X)=\sigma_{-}(X) \quad \text { for } X \text { without } \Gamma,
\end{array}\right.
\end{aligned}
$$

and these functions can be extended to $\mathrm{G}_{\gamma}$ since $\sigma \in \mathcal{B}_{\gamma}$.

By means of the substitutions

$$
\begin{aligned}
& F_{ \pm}=1+\varepsilon F_{ \pm}^{*} \\
& B_{ \pm}=\varepsilon B_{ \pm}^{*}, \\
& s_{1}=\varepsilon\left(F_{-}^{*}-F_{+}^{*}\right)+\varepsilon^{2} s_{1}^{*} \\
& s_{2}=\varepsilon\left(B_{+}^{*}-B_{-}^{*}\right)+\varepsilon^{2} s_{2}^{*} \\
& s_{3}=\varepsilon\left(B_{-}^{*}-B_{+}^{*}\right)+\varepsilon^{2} s_{3}^{*}
\end{aligned}
$$

the differential system (11) reads

$$
\frac{d}{d \varepsilon}\left(\begin{array}{c}
F_{+}^{*} \\
F_{-}^{*} \\
B_{+}^{*} \\
B_{-}^{*}
\end{array}\right)=\left(\begin{array}{c}
h_{1}\left(\varepsilon ; F_{+}^{*}, F_{-}^{*}, B_{+}^{*}, B_{-}^{*}\right) \\
h_{2}\left(\varepsilon ; F_{+}^{*}, F_{-}^{*}, B_{+}^{*}, B_{-}^{*}\right) \\
h_{3}\left(\varepsilon ; F_{+}^{*}, F_{-}^{*}, B_{+}^{*}, B_{-}^{*}\right) \\
h_{4}\left(\varepsilon ; F_{+}^{*}, F_{-}^{*}, B_{+}^{*}, B_{-}^{*}\right)
\end{array}\right)
$$

with the functions

$$
h_{j}:\left[0, \varepsilon_{\gamma}\right] \times \mathcal{B}_{\gamma}^{4} \rightarrow \mathcal{B}_{\gamma}
$$

satisfying a Lipschitz condition with respect to all arguments in a sufficiently small interval $\left[0, \varepsilon_{\gamma}\right]$; the upper limit $\varepsilon_{\gamma}$ is defined in this manner. Note that for the continuity of the linear operators $\hat{L}_{ \pm}$it is necessary to define them on $\mathcal{B}_{\gamma}$ and not on the Banach space of functions analytic at $\Gamma$.

As a consequence of the theorem by Picard and Lindelöff, the above system of differential equations has a solution which depends uniquely and continuously on the given initial conditions. This is the point at which we bring in the function $\gamma$ :

$$
F_{ \pm}^{*}(\varepsilon=0)=\hat{L}_{ \pm}\left(\gamma+\gamma^{*}\right), \quad B_{ \pm}^{*}(\varepsilon=0)=-\hat{L}_{ \pm}\left(\gamma-\gamma^{*}\right),
$$

where the function $\gamma^{*} \in \mathcal{B}_{\gamma}$ results from $\gamma$ by

$$
\gamma^{*}(X)=\overline{\gamma(\bar{X})} \quad \text { for } X \in \mathrm{G}_{\gamma} \text {. }
$$

These initial conditions together with the differential equations (11) yield $F_{ \pm}$and $B_{ \pm}$which only possess singularities without $(+)$ or within $(-)$ the curve $\Gamma$. Thus, the functions $F$ and $B$ obey a Riemann-Hilbert problem (10), and the associated functions $\alpha=\alpha(X ; \varepsilon ; \gamma)$ and $\beta=\beta(X ; \varepsilon ; \gamma)$ are uniquely determined. Consequently, we find a formulation $f=f(\varepsilon ; \gamma)$ by solving the linear integral equation that has been mentioned in (a) for ${ }^{6} \alpha=\alpha(X ; \varepsilon ; \gamma)$ and $\beta=\beta(X ; \varepsilon ; \gamma)$.

(c) If we prescribe $\gamma_{p}$ in the form

$$
\gamma_{p}(X)=H_{p}(X) \prod_{\nu=1}^{p}\left(X-X_{\nu}^{(p)}\right)
$$

then it can be shown that the Ernst potential following from the above reads

$$
f\left(\varepsilon ; \gamma_{p}\right)=f\left(\left\{X_{\nu}^{(p)}\right\}_{p}, \varepsilon H_{p}\right) .
$$

The proof of this uses many of the solution methods that were developed by Neugebauer and Meinel when they solved the boundary value problem of the rigidly rotating disk of dust. These methods are partially given in [29]; in full they will be treated in a subsequent paper.

For any series $\left\{\gamma_{p}\right\}_{p_{0}}^{\infty}$ of the above functions $\gamma_{p}$ with $\gamma_{p}$ $\rightarrow \gamma$ as $p \rightarrow \infty$ and $\gamma_{p} \in \mathcal{B}_{\gamma}$, the corresponding Ernst potentials $f=f\left(\varepsilon ; \gamma_{p}\right)$ converge for sufficiently small $\varepsilon$ since the

\footnotetext{
${ }^{6}$ The solubility of this integral equation is ensured for sufficiently small $\varepsilon$, see point (2) of the proof.
} 
functional $f=f(\varepsilon ; \gamma)$ depends continuously on $\gamma$. Because of the above equality, this implies the convergence of $f\left(\left\{X_{\nu}^{(p)}\right\}_{p}, \varepsilon H_{p}\right)$ as $p \rightarrow \infty$.

Remark. The differential system (11) can be explicitly solved if $\gamma$ is regular without $\Gamma$ with $\gamma \rightarrow 0$ as $|X| \rightarrow \infty$. Then one obtains

$$
\begin{aligned}
& \alpha=\cosh G+\left(\gamma+\gamma^{*}\right) \frac{\sinh G}{2 G}, \\
& \beta=-\left(\gamma-\gamma^{*}\right) \frac{\sinh G}{2 G}, \quad G^{2}=\gamma \gamma^{*},
\end{aligned}
$$

from which the Ernst potential along the rotation axis can be taken directly; see Eq. (13) below.

(2) The proof of the second statement uses the relations $\alpha=\alpha(X ; \varepsilon ; \gamma)$ and $\beta=\beta(X ; \varepsilon ; \gamma)$. In particular, we have $\alpha, \beta \in \mathcal{B}_{\gamma}$ for $\varepsilon \in\left[0, \varepsilon_{\gamma}\right]$ and moreover $\alpha=1+\mathcal{O}(\varepsilon)$ and $\beta$ $=\mathcal{O}(\varepsilon)$. This means that for sufficiently small $\varepsilon$, the linear integral equation that yields the Ernst potential from the functions $\alpha$ and $\beta$ can be solved for arbitrary coordinates $(\rho, \zeta) \notin B$; see [28]. Moreover, the Ernst potential can be extended to the region $\mathbb{B}_{\gamma}$ since $\alpha, \beta \in \mathcal{B} \gamma$. The different boundary values at the inner and outer sides of $B$ follow from the construction.

(3) Finally, the proof of the third statement uses the linear system (8). For $X \in \Gamma$ with $\Re X \geqslant 0$, we establish the matrices $\widetilde{\Phi}^{ \pm}\left[X, \rho=0, \zeta=r_{B}(1) \pm 0\right]$ by integrating the linear system along the inner and outer side of $B$, with the initial values

$$
\widetilde{\Phi}^{ \pm}(X, \Re X, \Im X)=\left(\begin{array}{cc}
1 & 1 \\
1 & -1
\end{array}\right) .
$$

The coordinate $\zeta=r_{B}(1) \pm 0$ stands for the inner and outer side of $B$ at $\rho=0$, respectively. The integration of the linear system can be performed since for a sufficiently weak relativistic regular Ernst potential again the theorem by Picard and Lindelöff applies. At $\rho=0$ and $\zeta=r_{B}(1) \pm 0$, we then establish $\Phi$ from $\widetilde{\Phi}$ by $\Phi=\widetilde{\Phi} M_{ \pm}$where the regular matrices $M_{+}$(defined on $\Gamma$ ) are chosen such that $\Phi$ assumes the structure (9), i.e. we calculate the functions $F_{ \pm}$and $B_{ \pm}$. In order to determine $\gamma$ we choose some $\varepsilon_{0}>0$ (say $\varepsilon_{0}=1$ ) and integrate the differential system (11) backwards, starting at $\varepsilon_{0}$ (here the initial conditions are just the established $F_{ \pm}, B_{ \pm}$), until we reach $\varepsilon=0$, and read $\gamma$ from the weak field expansion:

$$
\gamma=\lim _{\varepsilon \rightarrow 0} \frac{1}{2 \varepsilon}\left[\left(F_{-}-F_{+}\right)+\left(B_{+}-B_{-}\right)\right] .
$$

So we have proven that the interior and exterior boundary data $f$ and $f_{, z}, f_{, z}$ (the derivatives also enter the linear system) of a sufficiently weak relativistic Ernst potential, which is regular within and without $B$, uniquely determine the func-

\footnotetext{
${ }^{7}$ Note that the linear integral equation might globally only be soluble for $\varepsilon<\varepsilon_{\gamma}^{*}<\varepsilon_{\gamma}$.
}

tion $\gamma$. But since the weakly relativistic Dirichlet boundary value problem is uniquely soluble [24], the global interior and exterior Ernst potential (and hence the above derivatives) are determined by the boundary values $f$ alone, which therefore determines $\gamma$ as well.

\section{Properties of the generalized hyperelliptic solutions}

(1) For $\psi \in \mathbb{R}$ one finds

$$
f\left(e^{2 \mathrm{i} \psi} \varepsilon \gamma\right)=\frac{f(\varepsilon \gamma) \cos \psi+\mathrm{i} \sin \psi}{\cos \psi+\mathrm{i} f(\varepsilon \gamma) \sin \psi} .
$$

This can be seen by investigating $f\left(\left\{X_{\nu}\right\}_{p+1}, H_{R}\right)$ as $R \rightarrow \infty$ with

$$
\begin{aligned}
\gamma_{p}(X) & =H(X) \prod_{\nu=1}^{p}\left(X-X_{\nu}\right), \\
e^{2 \mathrm{i} \psi} \gamma_{p}(X) & =\lim _{R \rightarrow \infty} \gamma_{p+1} \\
& =\lim _{R \rightarrow \infty}\left[-\frac{H(X)}{R}\left(X-R e^{2 \mathrm{i} \psi}\right) \prod_{\nu=1}^{p}\left(X-X_{\nu}\right)\right] \\
& =\lim _{R \rightarrow \infty}\left[H_{R}(X)\left(X-X_{p+1}\right) \prod_{\nu=1}^{p}\left(X-X_{\nu}\right)\right] .
\end{aligned}
$$

Note that in the integral terms of Eqs. (6),(7) for $\nu=p+1$ the substitution $X=X_{p+1}\left(1+t^{2}\right)$ is useful.

The property (12) describes the general invariance transformation of $f(\varepsilon \gamma)$ which retains the asymptotic flatness $(f$ $\rightarrow 1$ as $r \rightarrow \infty) . f\left(e^{2 \mathrm{i} \psi} \varepsilon \gamma\right)$ is obtained from $f(\varepsilon \gamma)$ as one performs the transformation (21) of [30] with the parameters $\alpha=\sin ^{-2} \psi, \beta=\gamma=-\cot \psi$ of that paper.

(2) A given function $\gamma: \Gamma \rightarrow \mathrm{C}$ can be represented in many ways by sequences $\left\{\left\{X_{\nu}^{(p)}\right\}_{p}\right\}_{p_{0}}^{\infty}$ and $\left\{H_{p}\right\}_{p_{0}}^{\infty}$. Consider the following example for a spherical boundary, $r_{B} \equiv 1$. Since $\gamma$ is analytic on $\Gamma$, it can be written as

$$
\gamma(X)=\sum_{j=1}^{\infty}\left(\gamma_{j}^{(+)} X^{j-1}+\gamma_{j}^{(-)} X^{-j}\right), \quad X \in \Gamma
$$

where

$$
\begin{aligned}
\gamma_{j}^{( \pm)} & = \pm \frac{2 j-1}{2} \int_{0}^{\pi} Q_{ \pm}(\cos \vartheta) P_{j-1}(\cos \vartheta) \sin \vartheta d \vartheta \\
Q_{ \pm}(\cos \vartheta) & =\lim _{r \rightarrow 1 \mp 0}\left[\frac{1}{2 \pi \mathrm{i}} \oint_{\Gamma} \frac{\gamma(X) d X}{W_{z}}\right] .
\end{aligned}
$$

For each $n \in \mathbb{N}$ we find a representation 


$$
\begin{aligned}
\gamma_{n}(X) & =\sum_{j=1}^{n}\left(\gamma_{j}^{(+)} X^{j-1}+\gamma_{j}^{(-)} X^{-j}\right) \\
& =R_{n} e^{2 \mathrm{i} \psi_{n}} X^{m_{n}} \prod_{\nu=1}^{p_{n}}\left(X-X_{\nu}^{(n)}\right) .
\end{aligned}
$$

Thus we can write

$$
\begin{aligned}
f(\varepsilon \gamma) & =\lim _{n \rightarrow \infty} f\left(\varepsilon \gamma_{n}\right) \\
& =\lim _{n \rightarrow \infty}\left[\frac{f\left(\left\{X_{\nu}^{(n)}\right\}_{p_{n}}, \varepsilon H_{n}\right) \cos \psi_{n}+\mathrm{i} \sin \psi_{n}}{\cos \psi_{n}+\mathrm{i} f\left(\left\{X_{\nu}^{(n)}\right\}_{p_{n}}, \varepsilon H_{n}\right) \sin \psi_{n}}\right]
\end{aligned}
$$

with $H_{n}(X)=R_{n} X^{m_{n}}$.

(3) If the analytic function $\gamma: \Gamma \rightarrow \mathbb{C}$ is regular outside $\Gamma$ and $\gamma \rightarrow 0$ as $|X| \rightarrow \infty$, then one finds $f(\rho, \zeta ; \varepsilon \gamma)=1$ within $B$ and

$$
\begin{gathered}
f\left(\rho=0, \zeta>r_{B}(1) ; \varepsilon \gamma\right)=\left.\frac{G-s_{-} \sinh G}{G \cosh G+s_{+} \sinh G}\right|_{X=\zeta} \\
f\left(\rho=0, \zeta<-r_{B}(-1) ; \varepsilon \gamma\right)=\left.\frac{G \cosh G+s_{+} \sinh G}{G-s_{-} \sinh G}\right|_{X=\zeta}
\end{gathered}
$$

with

$$
\begin{aligned}
s_{ \pm}(X) & =\frac{\varepsilon}{2}[\gamma(X) \pm \overline{\gamma(\bar{X})}] \\
G^{2}(X) & =s_{+}^{2}(X)-s_{-}^{2}(X), \quad X \text { without } \Gamma .
\end{aligned}
$$

Hence, in the case of a flat interior field there is an explicit relation of the function $\gamma$ and the values of the Ernst potential along the exterior part of the symmetry axis.

(4) The Ernst potential $f=f(\varepsilon \gamma)$ possesses a reflectional symmetry

$$
f(\rho,-\zeta ; \varepsilon \gamma)= \begin{cases}\overline{f(\rho, \zeta ; \varepsilon \gamma)^{\kappa}} & \text { for }(\rho, \zeta) \text { within } B, \\ \overline{f(\rho, \zeta ; \varepsilon \gamma)}^{-\kappa} & \text { for }(\rho, \zeta) \text { without } B\end{cases}
$$

if $r_{B}(\tau)=r_{B}(-\tau)$ and $\overline{\gamma(-\bar{X})}=\kappa \gamma(X), \kappa^{2}=1$. We obtain the desired reflectional symmetry of the exterior Ernst potential for $\kappa=-1$.

(5) There is a weak field expansion of $f=f(\varepsilon \gamma)$ :

$$
\ln [f(\varepsilon \gamma)]=\frac{\varepsilon}{2 \pi \mathrm{i}} \oint_{\Gamma} \frac{\gamma(X) d X}{W_{z}}+\mathcal{O}\left(\varepsilon^{3}\right) .
$$

By differentiating Eqs. (6),(7) with respect to $\varepsilon$, where $H$ and $\left\{X_{\nu}\right\}_{p}$ are fixed, one deduces that

$$
\frac{d}{d \varepsilon} \ln \left[f\left(\left\{X_{\nu}\right\}_{p}, \varepsilon H\right)\right]=\frac{1}{2 \pi \mathrm{i}} \oint_{\Gamma} H(X) \prod_{\nu=1}^{p}\left(X-X^{(\nu)}\right) \frac{d X}{W_{z}} .
$$

The expansion (15) follows since, in the weak relativistic region, Eq. (7) yields $X^{(\nu)}=X_{\nu}+\mathcal{O}\left(\varepsilon^{2}\right)$.

(6) Another interesting property is

$$
f\left(\varepsilon \gamma^{*}\right)=\overline{f(\varepsilon \gamma)} \quad \text { for } \quad \gamma^{*}(X)=\overline{\gamma(\bar{X})}
$$

If, in particular, $\gamma=\gamma^{*}$ then $f=f(\varepsilon \gamma)$ is real and belongs to the Weyl class.

\section{APPROXIMATION OF ARBITRARY EXTERIOR BOUNDARY VALUE PROBLEMS BY BÄCKLUND TYPE SOLUTIONS}

Although the generalized hyperelliptic class permits the simultaneous solution of a sufficiently weak relativistic exterior and interior boundary value problem of the Ernst equation, its mathematical complexity makes it inconvenient for usage in a procedure to approximate the solution of the boundary value problem in question. Therefore one is led to investigate whether the much simpler Bäcklund type solutions suffice for our approximation scheme of only exterior boundary value problems. As we will demonstrate in this section, the freedom of the choice of the interior field allows us to set $\gamma \neq 0$ for $X \in \Gamma$, which ensures that the corresponding Ernst potential $f$ can be very well approximated by the Bäcklund type solutions.

\section{A. The class of the Bäcklund type solutions and its generalization}

\section{Definition}

The Bäcklund type solutions $f_{B}\left(\left\{Y_{\nu}\right\}_{q}, G\right)$ depend on the set $\left\{Y_{\nu}\right\}_{q}$ of complex parameters and on the analytic function $G: \Gamma \rightarrow \mathrm{C}$ with $\overline{G(X)}=G(\bar{X})$ and are defined by

$$
f_{B}\left(\rho, \zeta ;\left\{Y_{\nu}\right\}_{q}, G\right)=f_{0} \frac{D_{+}}{D_{-}}
$$

where

$$
\begin{aligned}
D_{ \pm} & =\left|\begin{array}{ccccc}
1 & 1 & 1 & \cdots & 1 \\
\pm 1 & \alpha_{1} \lambda_{1} & \alpha_{2} \lambda_{2} & \cdots & \alpha_{2 q} \lambda_{2 q} \\
1 & \lambda_{1}^{2} & \lambda_{2}^{2} & \cdots & \lambda_{2 q}^{2} \\
\pm 1 & \alpha_{1} \lambda_{1}^{3} & \alpha_{2} \lambda_{2}^{3} & \cdots & \alpha_{2 q} \lambda_{2 q}^{3} \\
\vdots & \vdots & \vdots & \ddots & \vdots \\
\pm 1 & \alpha_{1} \lambda_{1}^{2 q-1} & \alpha_{2} \lambda_{2}^{2 q-1} & \cdots & \alpha_{2 q} \lambda_{2 q}^{2 q-1} \\
1 & \lambda_{1}^{2 q} & \lambda_{2}^{2 q} & \cdots & \lambda_{2 q}^{2 q}
\end{array}\right|, \\
f_{0} & =\exp \left(\frac{1}{2 \pi \mathrm{i}} \oint_{\Gamma} \frac{G(X) d X}{W_{z}}\right), \\
\lambda_{2 \nu-1} & \sqrt{\frac{Y_{\nu}-\mathrm{i} \bar{z}}{Y_{\nu}+\mathrm{i} z}}, \quad \lambda_{2 \nu} \bar{\lambda}_{2 \nu-1}=1,
\end{aligned}
$$




$$
\begin{gathered}
\alpha_{2 \nu-1}=-\tanh \left(\frac{\lambda_{2 \nu-1}\left(Y_{\nu}+\mathrm{i} z\right)}{4 \pi \mathrm{i}} \oint_{\Gamma} \frac{G(X) d X}{\left(X-Y_{\nu}\right) W_{z}}\right), \\
\alpha_{2 \nu} \bar{\alpha}_{2 \nu-1}=1 .
\end{gathered}
$$

The Ernst potential $f_{B}\left(\left\{Y_{\nu}\right\}_{q}, G\right)$ is only then regular inside and outside the boundary $B$ if we additionally require that for each $Y_{\nu} \in \Gamma$ there is $G\left(Y_{\nu}\right)=0{ }^{8}$ For the desired procedure for approximating the solutions of exterior boundary value problems we will restrict ourselves to potentials $f_{B}\left(\left\{Y_{\nu}\right\}_{q}, G\right)$ with all $Y_{\nu} \notin \Gamma$.

\section{Bäcklund type solutions as special hyperelliptic solutions}

The Bäcklund type solutions form a subclass of the hyperelliptic solutions. In particular:

$$
f_{B}\left(\left\{Y_{\nu}\right\}_{q}, G\right)=f\left(\left\{Y_{1}, Y_{1}, \ldots, Y_{q}, Y_{q}\right\}, H\right)
$$

with

$$
H(X)=G(X)\left[\prod_{\nu=1}^{q}\left(X-Y_{\nu}\right)\left(X-\bar{Y}_{\nu}\right)\right]^{-1}
$$

and $f\left(\left\{X_{\nu}\right\}_{p}, H\right)$ as defined in Eq. (6). The proof of this works in the same manner as demonstrated in [23] for the solutions corresponding to disk-like sources. The regularity of the solutions $f\left(\left\{X_{\nu}\right\}_{p}, H\right)$ at $(\rho, \zeta)=\left(\left|\mathfrak{I}\left[X_{\nu}\right]\right|, \Re\left[X_{\nu}\right]\right)$ applies in this specialization and is exhibited by the fact that $\alpha_{j} \lambda_{j}$ is an even function in $\lambda_{j}$. This means that $f$ does not behave like a square root function near the critical points $(\rho, \zeta)=\left(\left|\Im\left[Y_{\nu}\right]\right|, \mathfrak{R}\left[Y_{\nu}\right]\right)$ but rather like a rational function.

\section{Bäcklund transformations}

The Ernst potentials (16) are special Bäcklund transformations as described in [28] [see formula (76)] with complex conjugate parameters $K_{2 \nu-1}=Y_{\nu}, K_{2 \nu}=\bar{Y}_{\nu}$ and the real seed solution $f_{0}=\exp [U(G)]$. The $\alpha_{j}$ in formula (76) of [28] satisfy the Riccati equations

$$
\alpha_{j, z}=\lambda_{j}\left(1-\alpha_{j}^{2}\right) \frac{f_{0, z}}{2 f_{0}}, \quad \alpha_{j, z}=\frac{1}{\lambda_{j}}\left(1-\alpha_{j}^{2}\right) \frac{f_{0, \bar{z}}}{2 f_{0}} .
$$

With constants $C_{j}$ of integration, the general solution of these equations reads

$$
\alpha_{j}=-\tanh \left(\frac{\lambda_{j}\left(K_{j}+\mathrm{i} z\right)}{4 \pi \mathrm{i}} \oint_{\Gamma} \frac{G(X) d X}{\left(X-K_{j}\right) W_{z}}+C_{j}\right) .
$$

By the particular choice in Eq. (16) [i.e. $C_{2 \nu-1}=0, C_{2 \nu}$ $=\mathrm{i} \pi / 2]$, the $\alpha_{j}$ become odd functions in $\lambda_{j}$ which ensures the regularity of the resulting Ernst potential at $(\rho, \zeta)$ $=\left(\left|\Im\left[Y_{\nu}\right]\right|, \mathfrak{R}\left[Y_{\nu}\right]\right)$.

\footnotetext{
${ }^{8}$ More precisely, if $\left|G\left(Y_{\nu}\right)\right|>\delta>0$ always holds, then the interior (exterior) Ernst potential encounters a square-root-like behavior at $(\rho, \zeta)=\left(\left|\Im\left[Y_{\nu}\right]\right|, \mathfrak{R}\left[Y_{\nu}\right]\right)$ when $Y_{\nu}$ tends to a point at the outer (inner) side of $\Gamma$.
}

\section{The generalization of the Bäcklund type solutions}

The function $\gamma_{q}$ which belongs to the Ernst potential $f$ $=f_{B}\left(\left\{Y_{\nu}\right\}_{q}, G\right)$ can be taken from Eq. (17):

$$
\gamma_{q}(X)=G(X) \prod_{\nu=1}^{q} \frac{X-Y_{\nu}}{X-\bar{Y}_{\nu}}
$$

It is therefore a consequence of the above theorem that for given analytic functions $G: \Gamma \rightarrow \mathrm{C}$ and $\Xi: \Gamma \rightarrow \mathbb{C}$ with $\overline{G(X)}$ $=G(\bar{X})$ and $\bar{\Xi}(X)=-\Xi(\bar{X})$, as well as sufficiently small $\varepsilon$, the Ernst potential

$$
f_{B}(\Xi, \varepsilon G)=\lim _{q \rightarrow \infty} f_{B}\left(\left\{Y_{\nu}^{(q)}\right\}_{q}, \varepsilon G\right)
$$

exists and is independent of the particular choice of the sequence $\left\{\left\{Y_{\nu}^{(q)}\right\}_{q}\right\}_{q_{0}}^{\infty}$ which serves to represent $\Xi$ by

$$
\Xi(X)=\lim _{q \rightarrow \infty} \ln \left[\prod_{\nu=1}^{q} \frac{X-Y_{\nu}^{(q)}}{X-\bar{Y}_{\nu}^{(q)}}\right] \quad \text { for } \quad X \in \mathbb{G}_{G} \cap \mathrm{G}_{\Xi} .
$$

In particular we have

$$
f_{B}(\Xi, \varepsilon G)=f(\varepsilon \gamma) \quad \text { with } \quad \gamma(X)=G(X) \exp [\Xi(X)] .
$$

From Eq. (18) we may conclude that any analytic function $\gamma$ which possesses a single zero $X_{\nu} \in \Gamma$ and does not vanish at $\bar{X}_{\nu}$ cannot be represented by two analytic functions $G$ and $\Xi$. Therefore, in the following we will restrict the discussion to $\gamma \neq 0$ for $X \in \Gamma$.

Remark. As a particular consequence we now can confirm the conjectures formulated in [23], Secs. 2 and 5. The first one of these conjectures treats the generalization of Bäcklund type solutions that describe disk-like sources of the gravitational field. In order to map the treatment of that place to our extended $B$ here, we consider for given functions ${ }^{9} \xi$ and $g$ and an arbitrary point $(\rho, \zeta)$ without the disk, a boundary $B$ which encompasses the disk but leaves the point $(\rho, \zeta)$ outside $B$ as well as all singularities of $g\left(-X^{2} / \rho_{0}^{2}\right)$ and $\xi\left(-X^{2} / \rho_{0}^{2}\right)$ outside $\Gamma\left(\rho_{0}\right.$ is the radius of the disk). If we now take any sequence $\left\{\left\{Y_{\nu}^{(q)}\right\}_{q}\right\}_{q_{0}}^{\infty}$ which serves to represent $\xi$ by

$$
\begin{aligned}
\xi\left(x^{2}\right) & =\lim _{q \rightarrow \infty} \xi_{q}\left(x^{2}\right) \\
\xi_{q}\left(x^{2}\right) & =\frac{1}{x} \ln \left[\prod_{\nu=1}^{q} \frac{\mathrm{i} Y_{\nu}^{(q)}-x}{\mathrm{i} Y_{\nu}^{(q)}+x}\right] \quad \text { for } \quad x \in[-1,1]
\end{aligned}
$$

with all $\left(\rho_{0} Y_{\nu}^{(q)}\right)$ being outside $\Gamma$, then the resulting Ernst potential $f\left(\rho, \zeta ; \xi_{q}, g\right)$ in [23] at the chosen point $(\rho, \zeta)$ coincides with the above $f_{B}\left(\rho, \zeta ; \Xi_{q}, G_{q}\right)$ when

\footnotetext{
${ }^{9}$ In [23], $\xi$ and $g$ were assumed to be real analytic functions defined on the interval $[0,1] . g$ describes the seed solution while $\xi$ comprises the Bäcklund parameters.
} 


$$
\begin{aligned}
G_{q}\left(\mathrm{i} \rho_{0} x\right) & =2(-1)^{q+1} \operatorname{arccot}(\mathrm{i} x) g\left(x^{2}\right), \\
\exp \left[\Xi_{q}\left(\mathrm{i} \rho_{0} x\right)\right] & =(-1)^{q} \exp \left[-x \xi_{q}\left(x^{2}\right)\right] .
\end{aligned}
$$

As $q \rightarrow \infty$, the corresponding functions $\gamma_{q}$ with

$$
\gamma_{q}\left(\mathrm{i} \rho_{0} x\right)=G_{q}\left(\mathrm{i} \rho_{0} x\right) \exp \left[\Xi_{q}\left(\mathrm{i} \rho_{0} x\right)\right]
$$

converge towards the function $\gamma$,

$$
\gamma\left(\mathrm{i} \rho_{0} x\right)=-2 \operatorname{arccot}(\mathrm{i} x) g\left(x^{2}\right) \exp \left[-x \xi\left(x^{2}\right)\right] .
$$

So, due to the above theorem, the Ernst potentials $f\left(\rho, \zeta ; \xi_{q}, g\right)$ in $[23]$ tend to $f(\rho, \zeta ; \gamma)$. Hence we can conclude, that the exterior gravitational field of a disk-like source is determined only by the local functions $g$ and $\xi$, and does not depend on a particular global representation of $\xi$ in terms of the parameters $Y_{\nu}$. This is just the statement of the assumption in Sec. 2 of [23]. Note that the condition that $Y_{\nu}$ be outside the imaginary interval $[-\mathrm{i}, \mathrm{i}]$ is always realized for sufficiently weak relativistic differentially rotating disks of dust since this is ensured by the positivity of the surface mass density $\sigma_{p}$ (see Appendix C.1.2 of [23]; the function $g_{0}$ is strictly positive and therefore the function $\xi_{0}$ is analytic). As one moves to more relativistic disks one might encounter a situation in which $g$ and $\xi$ are not analytic (corresponding to the situation discussed above). However, for the examples investigated, this situation did not occur.

In Sec. 5 of [23], conjectures were given regarding the functions $g$ and $\xi$ for the disk-like hyperelliptic class of solutions. These conjectures are also proven by the above considerations. A specially chosen disk-like hyperelliptic solution as presented in Appendix A of [23] with an analytic function $h$ and parameters $\left\{X_{\nu}\right\}_{p}$, coincides with $f(\rho, \zeta ; \gamma)$ at a given point $(\rho, \zeta)$ outside the disk, when $\gamma$ reads

$$
\begin{aligned}
\gamma\left(\mathrm{i} \rho_{0} x\right) & =-2 \operatorname{arccot}(\mathrm{i} x) \gamma_{D}\left(\mathrm{i} \rho_{0} x\right), \\
\gamma_{D}\left(\mathrm{i} \rho_{0} x\right) & =h\left(x^{2}\right) \prod_{\nu=1}^{p}\left(\mathrm{i} x-X_{\nu}\right)
\end{aligned}
$$

and a corresponding boundary $B$ is taken in the above manner with the singularities of $\gamma_{D}$ being outside $\Gamma$. The comparison with Eq. (19) yields exactly the conjectured functions $g$ and $\xi$ in terms of $h$ and $\left\{X_{\nu}\right\}_{p}$. It is of particular interest that thus the Neugebauer-Meinel-solution can be written as a well defined limit of Bäcklund type solutions.

\section{B. The approximation scheme and results}

In this section we describe our procedure to approximate the solution to an exterior boundary value problem of the Ernst equation. Due to the preceding formulations, we seek an appropriate function $\gamma$ such that the corresponding Ernst potential $f=f(\gamma)$ assumes prescribed exterior boundary values at $B$. As outlined above, we only consider functions $\gamma$ that do not vanish at $\Gamma$, and for which the corresponding Ernst potentials can be well approximated by the Bäcklund type solutions. We find in particular that in contrast to the Laplace equation, arbitrary boundary values of the Dirichlet type are not possible.

\section{The approximation scheme}

(1) At first we restrict the function $\gamma$ in an appropriate manner. Because of the desired reflectional symmetry (14) we take $r_{B}(\tau)=r_{B}(-\tau)$ and $\overline{\gamma(-\bar{X})}=-\gamma(X)$. To avoid zeros at $\Gamma$ we demand $\mathfrak{I}(\gamma)=$ const $=\gamma_{0} \neq 0$ for $X \in \Gamma$. This leads to

$$
\gamma(X)=\mathrm{i} \gamma_{0}+A_{1}(X)+A_{2}(X)
$$

with

$$
\begin{aligned}
A_{1}\left[X=r_{B}(\cos \theta) e^{\mathrm{i} \theta}\right] & =\sum_{k=1}^{\infty} \gamma_{2 k-1} \cos [(2 k-1) \theta], \\
A_{2}\left[X=r_{B}(\cos \theta) e^{\mathrm{i} \theta}\right] & =\sum_{k=1}^{\infty} \gamma_{2 k} \sin [2 k \theta], \\
\gamma_{j} & \in R, \quad \theta \in[0,2 \pi] .
\end{aligned}
$$

The real constant $\gamma_{0} \neq 0$ may be chosen arbitrarily; the coefficients $\gamma_{j}$ for $j \geqslant 1$ are then determined uniquely by the exterior boundary conditions.

(2) It is now possible to find analytic functions $\hat{G}$ and $\hat{\Xi}$ with

$$
\begin{aligned}
& \gamma(X)= \mathrm{i} \hat{\gamma}(X)=\mathrm{i} \hat{G}(X) \exp [\hat{\Xi}(X)], \\
& \hat{G}(X)= \sqrt{A_{1}^{2}(X)-\left[A_{2}(X)+\mathrm{i} \gamma_{0}\right]^{2}}, \\
& \hat{G}\left[X=\mathrm{i} r_{B}(0)\right]=\gamma_{0}, \\
& \hat{\Xi}(X)=-\frac{\mathrm{i}}{2}\left[\arctan \left(\frac{A_{1}(X)+A_{2}(X)}{\gamma_{0}}\right)\right. \\
&\left.+\arctan \left(\frac{A_{1}(X)-A_{2}(X)}{\gamma_{0}}\right)\right] \\
&+\frac{1}{4} \ln \left[\frac{\left[A_{1}(X)+A_{2}(X)\right]^{2}+\gamma_{0}^{2}}{\left[A_{1}(X)-A_{2}(X)\right]^{2}+\gamma_{0}^{2}}\right], \\
& \hat{\Xi}\left[X=\mathrm{i} r_{B}(0)\right]=0 .
\end{aligned}
$$

With Eq. (12), the Ernst potential reads then as follows:

$$
f(\gamma)=\frac{f_{B}(\hat{\Xi}, \hat{G})+\mathrm{i}}{1+\mathrm{i} f_{B}(\hat{\Xi}, \hat{G})} .
$$

(3) For a given analytic function $\hat{\Xi}$ of the above kind we determine corresponding Bäcklund parameters $\left\{Y_{\nu}\right\}_{q}$ in the same manner as outlined in [23]. This means that we solve the linear system: 


$$
\begin{aligned}
& \exp \left[\hat{\Xi}\left(Z_{\nu}\right)\right] P\left(-Z_{\nu}\right)=(-1)^{q} P\left(Z_{\nu}\right), \quad \nu=1 \ldots q \\
& P(X)=\prod_{\nu=1}^{q}\left(X-Y_{\nu}\right)=X^{q}+\sum_{j=0}^{q-1} b_{j} X^{j}
\end{aligned}
$$

to determine the coefficients $b_{j}$ and from these the zeros $Y_{\nu}$ of $P$. The $q$ arbitrary different supporting points $Z_{\nu} \in \Gamma$ are chosen to possess positive real and imaginary parts.

(4) By virtue of formula (16) we are now able to approximate the Ernst potential $f(\gamma)$ if the parameters $\left\{\gamma_{j}\right\}_{0}^{\infty}$ are given. For the approximate numerical evaluation of $\left\{\gamma_{j}\right\}_{1}^{2 n}$ from the given exterior boundary values we set $\gamma_{j}=0$ for $j$ $>2 n$ and demand that the resulting exterior Ernst potential $f(\gamma)$ coincide with the prescribed boundary values at $n$ different points $\left(\rho_{j}, \zeta_{j}\right) \in B$ with $\zeta_{j} \geqslant 0$. This gives a complicated nonlinear set of $2 n$ real equations to determine the unknown coefficients $\left\{\gamma_{j}\right\}_{1}^{2 n}$. As in the analogous treatment to solve boundary value problems for arbitrary differentially rotating disks of dust, we solve this system by means of a Newton-Raphson method. Again we provide good initial guesses by solving a sequence of boundary value problems with initially weak relativistic and finally the desired boundary values, and where the initial guess comes from the weak relativistic expansion (15).

\section{Results}

The above approximation scheme has been executed for various Dirichlet boundary value problems. As expected from the above, there is always a weak relativistic region within which the solution exists and can be well approximated by the Bäcklund type solutions. In particular, the exterior solution proves to be independent of the choice of the parameter $\gamma_{0}$, only the accuracy of the results is affected.

As one moves further to more relativistic boundary values, there might be however a limit beyond which the solutions cannot be extended. For example, take the exterior boundary values

$$
f[(\rho, \zeta) \in B]=1+\varepsilon\left[-\frac{3}{2}+\zeta^{2}\right]+\mathrm{i} \varepsilon \zeta,
$$

with the spherical boundary $B$ characterized by $r_{B} \equiv 1$. One finds a limiting parameter $\varepsilon_{0} \approx 0.68$ beyond which the boundary value problem does not seem to be soluble. This has been verified not only by various choices of the above parameter $\gamma_{0}$ but also by a two-dimensional numerical method, which solves the exterior Ernst equation directly without using analytic solutions. ${ }^{10}$ All these routines yield the same limiting parameter $\varepsilon_{0}$ which is a strong indication that for $\varepsilon>\varepsilon_{0}$ the Ernst potentials corresponding to the above boundary values do not exist.

\section{ACKNOWLEDGMENTS}

The support from the DFG is gratefully acknowledged.

\footnotetext{
${ }^{10}$ For this method, we insert a two-dimensional Chebyshev expansion of the exterior Ernst potential into the Ernst equation and find the Chebyshev coefficients.
}

[1] F. J. Ernst, Phys. Rev. 167, 1175 (1968).

[2] D. Kramer and G. Neugebauer, Commun. Math. Phys. 7, 173 (1968).

[3] D. Maison, Phys. Rev. Lett. 41, 521 (1978).

[4] V. A. Belinski and V. E. Zakharov, Zh. Eksp. Teor. Fiz. 75, 195 (1978).

[5] B. K. Harrison, Phys. Rev. Lett. 41, 1197 (1978).

[6] E. Herlt, Gen. Relativ. Gravit. 9, 711 (1978).

[7] C. Hoenselaers, W. Kinnersley, and B. C. Xanthopoulos, Phys. Rev. Lett. 42, 481 (1979).

[8] G. Neugebauer, J. Phys. A 12, L67 (1979).

[9] G. Neugebauer, J. Phys. A 13, L19 (1980).

[10] G. Neugebauer, J. Phys. A 13, 1737 (1980).

[11] I. Hauser and F. J. Ernst, Phys. Rev. D 20, 362 (1979).

[12] I. Hauser and F. J. Ernst, Phys. Rev. D 20, 1783 (1979).

[13] I. Hauser and F. J. Ernst, J. Math. Phys. 21, 1418 (1980).

[14] G. Neugebauer and R. Meinel, Astrophys. J. Lett. 414, L97 (1993).

[15] G. Neugebauer and R. Meinel, Phys. Rev. Lett. 75, 3046 (1995).

[16] G. Neugebauer, A. Kleinwächter, and R. Meinel, Helv. Phys.
Acta 69, 472 (1996).

[17] R. Meinel and G. Neugebauer, Phys. Lett. A 210, 160 (1996).

[18] D. A. Korotkin, Theor. Math. Phys. 77, 1018 (1989).

[19] D. A. Korotkin, Class. Quantum Grav. 10, 2587 (1993).

[20] D. A. Korotkin, Phys. Lett. A 229, 195 (1997).

[21] R. Meinel and G. Neugebauer, Phys. Lett. A 229, 200 (1997).

[22] M. Ansorg and R. Meinel, Gen. Relativ. Gravit. 32, 1365 (2000).

[23] M. Ansorg, Gen. Relativ. Gravit. 33, 309 (2001).

[24] O. Reula, Commun. Math. Phys. 122, 615 (1989).

[25] U. M. Schaudt and H. Pfister, Phys. Rev. Lett. 77, 3284 (1996).

[26] U. M. Schaudt, Commun. Math. Phys. 190, 509 (1998).

[27] R. Meinel and G. Neugebauer, Class. Quantum Grav. 12, 2045 (1995).

[28] G. Neugebauer, in General Relativity, Proceedings of the 46th Scottish Universities Summer School in Physics, Aberdeen, 1995, edited by G. S. Hall and J. R. Pulham (The Institute of Physics, London, 1996), p. 61.

[29] G. Neugebauer, Ann. Phys. (Leipzig) 9, 342 (2000).

[30] D. Kramer and G. Neugebauer, Commun. Math. Phys. 10, 132 (1968). 